\title{
Sustained Complete Remission in Multi-Relapsed Primary CNS Lymphoma Treated with Ibrutinib Monotherapy: A Case Report
}

\author{
Anna Maira Armin Muigg ${ }^{b}$ Günther Stockhammer ${ }^{b}$ \\ Stephanie Mangesius ${ }^{c}$ Dominik Wolf ${ }^{a}$ Eberhard Gunsilius ${ }^{a}$ \\ aDepartment of Hematology \& Medical Oncology, Medical University Innsbruck, Innsbruck, \\ Austria; bepartment of Neurology, Medical University Innsbruck, Innsbruck, Austria; \\ 'Department of Neuroradiology, Neuroimaging core facility, Medical University Innsbruck, \\ Innsbruck, Austria
}

\section{Keywords}

Ibrutinib · Primary CNS lymphoma

\section{Abstract}

Primary CNS lymphoma (PCNSL) is a highly aggressive malignant disease with a high recurrence rate and a poor prognosis. We present the case of a 71-year-old woman diagnosed with PCNSL in June 2010. After 3 relapses and intensive treatment with multiple chemotherapy regimens and whole-brain radiotherapy, she received off-label treatment with the Bruton tyrosine kinase inhibitor ibrutinib, responded well, achieved a complete remission, and is progression-free for now $>3$ years.

\section{Background}

Primary CNS lymphoma (PCNSL) is a highly aggressive extranodal non-Hodgkin lymphoma presenting as infiltration of Epstein-Barr virus-negative lymphoma cells in the brain parenchyma, leptomeninges, spinal cord, or the eye without evidence of systemic disease [1]. In immunocompetent patients, PCNSL is a rare disease accounting for $3 \%$ of newly diagnosed primary central nervous system tumors and for $<3 \%$ of non-Hodgkin lymphomas, with a median age of 
64 years [2]. Unfavorable prognostic factors are age greater than 60 years, an Eastern Cooperative Oncology Group performance status greater than 1, elevated serum lactate dehydrogenase level, elevated cerebrospinal fluid protein concentration, and involvement of the deep regions of the brain (IELSG-score). In patients with $0 / 1$ factors, $2 / 3$ factors, $>3$ risk factors, the 2-year survival was $80 \%, 48 \%$, and $15 \%$, respectively [3]. Standard induction treatment contains highdose methotrexate (HD-MTX), the anti-CD20 antibody rituximab, and an alkylating agent with or without cytarabine followed by high-dose chemotherapy and autologous hematopoietic stem cell transplantation in younger patients. Although new therapeutic regimes have improved outcomes especially in younger patients, elderly patients with PCNSL still have a very poor prognosis, especially when relapsing after first-line therapy [1]. The survival after relapse is usually $<1$ year and currently no optimal treatment regimen exists for those patients [4].

\section{Case Presentation}

In June 2010, a 61-year-old woman presented to the emergency department with increased fatigue, mild expressive aphasia, and progressive homonymous hemianopia to the right side that progressed over 3 weeks. Brain MRI demonstrated a lesion in the left mesial occipital lobe suspicious for lymphoma. Stereotactic needle biopsy confirmed PCNSL, diffuse large B-cell type (KI67 100\% positive, CD20 and CD79 positive). No mutations in the CD79b or MYD88 genes were found by next-generation sequencing (performed 2021). No evidence of systemic spread was found on whole-body FDG-positron emission tomography/computed tomography and bone-marrow biopsy. No malignant cells were detected in the cerebrospinal fluid. The IELSG-score was low-risk. The treatment applied for induction consisted of 6 cycles of HD-MTX together with either ifosfamide (cycle 1 and 4), cyclophosphamide (cycle 2 and 5) or high-dose cytosine arabinoside (cycle 3 and 6) [5]. The disease responded promptly, and she achieved a complete remission in November 2010. She refused consolidation with highdose chemotherapy and autologous hematopoietic stem cell transplantation. For the first and second relapses occurring March 2013 and August 2016, 6 and 4 cycles of HD-MTX in combination with rituximab were applied, respectively. In total she received 14 courses of HD-MTX, the most active drug in PCNSL, with various combination partners. Furthermore, whole-brain radiotherapy (30,4 Gy, fractions of 1.6 Gy per day with a boost of 6,4 Gy to the lymphoma site) was applied from February 2017 to March 2017 for the treatment of the third relapse.

As in June 2017, Lionakis and colleagues reported the inhibition of B-cell receptor (BCR) signaling in PCNSL by ibrutinib and observed regression of PCNSL in 94\% of 18 patients in their phase-I clinical trial, we decided to administer off-label treatment with ibrutinib $560 \mathrm{mg}$ orally per day for treatment of the fourth recurrence in January 2018 [6]. Cerebral MRI 2 weeks later demonstrated tumor regression. An MRI after 10 months showed a complete response (Fig. 1) which is still ongoing (last MRI in March 2021). Treatment with ibrutinib in our patient was accompanied by infectious complications, such as repeated bacterial urogenital infections, an abscess in the floor of the mouth, a nosocomial pneumonia and recurrent genito-anal herpes simplex infections due to severe hypogammaglobulinemia with immunoglobulin-G serum levels below $100 \mathrm{mg} / \mathrm{dL}$, requiring intravenous acyclovir and regular prophylactic substitution of immunoglobulins.

\section{Discussion}

The prognosis of patients with relapsed or refractory PCNSL is dismal, with an overall survival time from relapse of usually $<1$ year, in which patients with longer first remission after initial HD-MTX treatment may benefit from re-exposure to this drug [7]. Ibrutinib is an 

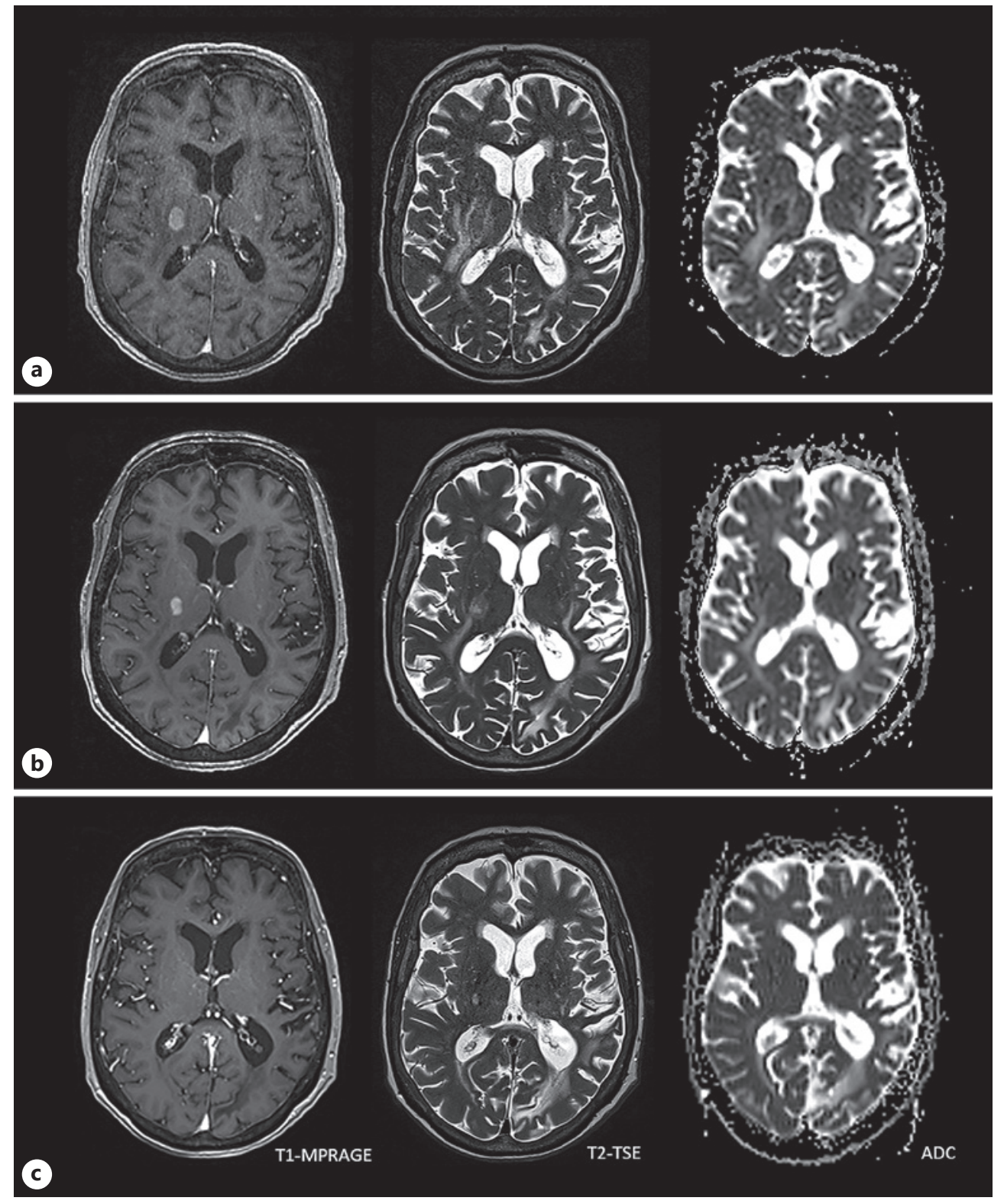

Fig. 1. MRI of the PCNSL (a) at fourth recurrence and (b) tumor regression 2 weeks and (c) l after off-label treatment with ibrutinib 560 mg orally per day. MR images: axial contrast-enhanced T1- MPRAGE sequences after intravenous application of $6 \mathrm{~mL}$ Gadovist, axial T2- TSE sequences, and axial ADC sequences acquired on a (a) 1,5 T (T) SymphonyTim SIEMENS and (b) 3 T Verio SIEMENS scanner. Image interpretation: (a) tumor is depicted as homogenously contrast-enhancing, to grey matter T2-hypointense, diffusion restricted (low ADC values) lesions (tumor recurrence). $\mathbf{b}$ Tumor is depicted as contrast-enhancing lesions of decreasing size, decreasing T2-hypointensity to partly T2-hyperintensity, and nearly to normal ADC values (tumor regression). c No contrast-enhancing or T2-hypointense lesions are depicted (complete remission). PCNL, primary CNS lymphoma; TSE, Turbo Spin Echo; ADC, Apparent Diffusion Coefficient; MPRAGE, Magnetization Prepared Rapid Acquisition with Gradient Echoes.

oral, first-in-class, small-molecule irreversible inhibitor of Bruton's tyrosine kinase and blocks NFkB activation downstream of the BCR signaling. Currently, ibrutinib is approved for the treatment of the B-cell malignancies mantle cell lymphoma, chronic lymphocytic leukemia, and Waldenstrom's macroglobulinemia.

Karger' 
The blood-brain barrier complicates the treatment of PCNSL, as multiple agents are unable of crossing it and therefore remain ineffective. Ibrutinib and its active metabolite PCI-45227 pass the blood-brain barrier and reach effective concentrations in cerebrospinal fluid [6].

Diffuse large B-cell lymphoma of the activated-B-cell type acquire mutations, for example, of the CD79b (BCR subunit) and/or MYD88 (toll-like receptor adaptor protein) that target the BCR, leading to chronic BCR signaling [8]. Likewise, PCNSL shows a high frequency of these mutations that augment BCR signaling, rendering them particularly sensitive to treatment with ibrutinib. In our case, none of these 2 mutations was detectable by next-generation sequencing, implicating that the lymphoma cells were anyway dependent on BCL-signaling. Thus, Ibrutinib treatment should not be restricted to mutated cases of PCNSL.

In a phase-I trial using up to $840 \mathrm{mg}$ ibrutinib daily, the clinical response rate was $77 \%$ ( $n=17,5$ complete remissions) [9]. Toxicity was mainly hematotoxicity and 1 pulmonary aspergillosis occurred. In a recent phase-II trial with 44 evaluable patients treated with $560 \mathrm{mg}$ ibrutinib daily for relapsed or refractory PCNSL, the response rates were $17 \%$ complete response, 33\% partial response, and 10\% stable disease, demonstrating the remarkable clinical efficacy of ibrutinib monotherapy in this patient group. After a median follow-up of 25,7 months, the progression-free and overall survival were 4,8 months and 19,2 months, respectively. Two patients experienced pulmonary aspergillosis, one with fatal outcome [10].

This case, to our knowledge one of the first PCNSL patients undergoing long-term treatment with ibrutinib achieving durable disease control, demonstrates in rare clarity the potential benefit of ibrutinib treatment in patients with multi-relapsed and heavily pretreated PCNSL with acceptable toxicity. Monitoring of serum immunoglobulins is required and the possible complication of invasive aspergillosis must be kept in mind.

\section{Statement of Ethics}

The work was approved by the Ethics Committee of the medical university Innsbruck (No $1030 / 2021)$. Written informed consent was obtained from the patient for publication of this case report.

\section{Conflict of Interest Statement}

The authors have no conflicts of interest to declare.

\section{Funding Sources}

No funding.

\section{Author Contributions}

A.M. and E.G. wrote the manuscript. A.M., G.S., E.G., and D.W. were responsible for the treatment of the patient and revised the manuscript. S.M. provided neuroradiological details and revised the manuscript. 


\section{Data Availability Statement}

The clinical data of this case report are available on request from the corresponding author.

\section{References}

1 Grommes C, Pastore A, Palaskas N, Tang SS, Campos C, Schartz D, et al. Ibrutinib unmasks critical role of Bruton tyrosine kinase in primary CNS lymphoma. Cancer Discov. 2017 Jul 20;7(21):1018-29.

2 Chukwueke UN, Nayak L. Central nervous system lymphoma. Hematol Oncol Clin North Am. 2019 Aug;33(4): 597-611.

3 Ferreri AJ, Blay JY, Reni M, Pasini F, Spina M, Ambrosetti A, et al. Prognostic scoring system for primary CNS lymphomas: the International Extranodal Lymphoma Study Group experience. J Clin Oncol. 2003 Jan 15;21(2): 266-72.

4 Bonm AV, Gibson AW, Holmberg LA, Mielcarek M, McGranahan T, Taylor LP, et al. A single-center retrospective analysis of outcome measures and consolidation strategies for relapsed and refractory primary CNS lymphoma. J Neurooncol. 2021 Jan;151(2):193-200.

5 Pels H, Schmidt-Wolf IG, Glasmacher A, Schulz H, Engert A, Diehl V, et al. Primary central nervous system lymphoma: results of a pilot and phase II study of systemic and intraventricular chemotherapy with deferred radiotherapy. J Clin Oncol. 2003 Dec 15;21(24):4489-95.

6 Lionakis MS, Dunleavy K, Roschewski M, Widemann BC, Butman JA, Schmitz R, et al. Inhibition of B cell receptor signaling by Ibrutinib in primary CNS lymphoma. Cancer cell. 2017 Jun 12;31(6):833-843.e5.

7 Schaff LR, Grommes C. Updates on primary central nervous system lymphoma. Curr Oncol Rep. 2018 Feb 28; 20(2):11.

8 Wilson WH, Young RM, Schmitz R, Yang Y, Pittaluga S, Wright G, et al. Targeting B cell receptor signaling with ibrutinib in diffuse large B cell lymphoma. Nat Med. 2015 Aug;21(8):922-6.

9 Grommes C, Pastore A, Palaskas N, Tang SS, Campos C, Schartz D, et al. Ibrutinib unmasks critical role of Bruton tyrosine kinase in primary CNS lymphoma. Cancer Discov. 2017 Sep;7(9):1018-29.

10 Soussain C, Choquet S, Blonski M, Leclercq D, Houillier C, Rezai K, et al. Ibrutinib monotherapy for relapse or refractory primary CNS lymphoma and primary vitreoretinal lymphoma: final analysis of the phase II 'proofof-concept' iLOC study by the Lymphoma study association (LYSA) and the French oculo-cerebral lymphoma (LOC) networkEur J Cancer. 2019;117:121-30. 